\section{WETLAND EMISSIONS}

\section{Methane transport in plants \\ Limnol. Oceanogr. 65, 1635-1650 (2020).}

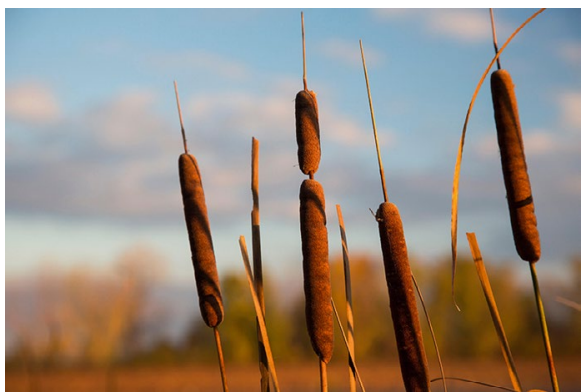

Credit: Jim West / Alamy Stock Photo

Wetlands are the largest natural source of methane to the atmosphere. In freshwater mineral-soil wetlands, about $30-90 \%$ of methane fluxes are mediated by plants through a reversal of mechanisms in place to transport oxygen into the roots as an adaptation to the predominantly anoxic conditions in wetland soils. The rates of methane transport by plants, regulated by photosynthesis and stomatal conductance, are highly variable and are not well represented in models due to a lack of observational data, leading to high variability in model results.

Jorge Villa from Ohio State University, USA, and colleagues investigate methane flux, plant-mediated methane transport and carbon uptake in three plant species (cattail, American lotus and water lily). They find that plant conductance of methane depends on the species as well as leaf area, and varies intra-seasonally. Although methane flux and $\mathrm{CO}_{2}$ uptake were correlated, this relationship cannot be generalized across plant functional types. Nevertheless, using species - distinguished based on whether gas transport is stomatal-controlled - could improve model predictions of wetland methane emissions.

https://doi.org/10.1038/s41558-020-0867-0

\section{STRESS RESPONSES}

\section{Roles of repetition and race}

BMC Genom. 21, 396 (2020).

Extreme climate-related events threaten food security. The predicted continued increase in frequency and severity of drought, a major contributor to crop losses, highlights the need to better understand plant drought responses as a first step in improving crop resilience.

Caitlin Simopoulos, Mitchell MacLeod and colleagues at McMaster University, Canada, investigated drought response in Eutrema salsugineum, a salt-tolerant extremophyte species closely related to cruciferous crops. Previous research comparing drought response in two ecotypes (races) of E. salsugineum, revealed that while the ecotypes responses were physically similar under a single drought treatment, they differed when the stress was repeated. Here, comparative transcriptomics was undertaken to clarify gene expression differences between the two ecotypes during a two-stage drought treatment. The study revealed extensive drought-related reprogramming that vastly differed between the ecotypes.

\title{
CLIMATE CHANGE ADAPTATION
}

\section{Limited media narratives}

Adaptation is necessary to minimize the harm caused by climate change and the associated increase in severity and frequency of extreme events. However, to develop adaptation strategies, decisions must be made about who should adapt, when, and for what purpose. Media coverage of adaptation may shape public opinion on these issues through narratives that are repeated and reinforced over time, and could ultimately inform whether societies choose to adapt, and how.

Rachel Harcourt and colleagues from University of Leeds and University of Southern California conducted a narrative analysis of adaptation coverage in national and regional UK newspapers. They found that media coverage tended to present adaptation as a response to immediate threats such as flooding, which are dependent on national government action and intended to preserve the current way of life. There was little discussion of long-term challenges and compromises. The authors caution that this narrow view of adaptation focussing on flooding and government response might undermine individual adaptation efforts, disengage those not facing flood risks and restrict the range of policy options under consideration.

https://doi.org/10.1038/s41558-020-0869-y

These works highlight the importance of understanding how organisms respond to successive (as opposed to single) stress events, and underscore how these responses can be driven by genetic diversity that is apparent even at the sub-species level. TAM

https://doi.org/10.1038/s41558-020-0870-5

\section{URBAN HEAT ISLANDS \\ Aerosol-driven seasonality}

Atmos. Chem. Phys. 20, 6479-6493 (2020).

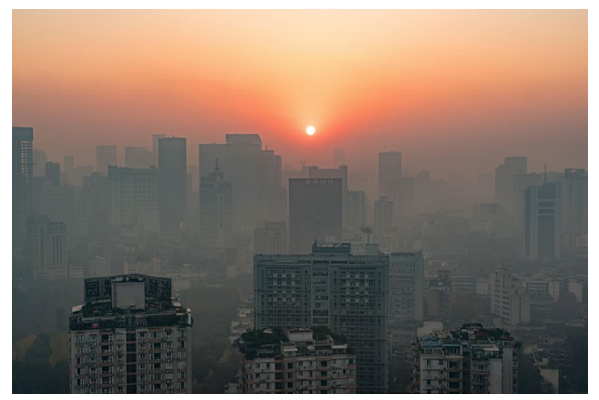

Credit: Imaginechina Limited / Alamy Stock Photo

City expansion alters the land surface and can cause an urban heat island (UHI), where a metropolitan area is warmer than its surroundings, particularly during the daytime and under weak winds. Cities are also known for their substantial aerosol emissions, generally thought to absorb and reflect sunlight, shade the land surface and offset daytime UHI intensity. However, these impacts can vary throughout the year, and seasonal differences are not well documented.

Wenchao Han from Beijing Normal University, China, and co-authors used satellite and ground-based observations for 35 Chinese cities during 2001-2010, as well as a regional climate model, to study the seasonality of aerosol-UHI interaction. During the summer, aerosols typically decrease UHI intensity, as expected from the aerosol radiative effect that shades the surface. In contrast, aerosol pollution tends to increase UHI intensity during the winter. The authors attribute this to an aerosol dynamic effect, where aerosols absorb sunlight aloft and stabilize the atmosphere, preventing vertical mixing and cooling. These results add critical understanding to the interactions between urban development, air pollution and heat exposure. 